\title{
Optimized temperature/bandwidth operation of cryogenic Yb:YAG composite thin-disk laser amplifier
}

\author{
Anne-Laure Calendron ${ }^{1,2}$, Luis E. Zapata ${ }^{3}$, Hüseyin Çankaya ${ }^{1,2}$, \\ Hua Lin $^{3}$ and Franz X. Kärtner ${ }^{1,2,3}$ \\ ${ }^{1}$ Center for Free-Electron Laser Science, Deutsches Elektronen Synchrotron, and Department of Physics, University of Hamburg, Notkestrasse \\ 85, 22607 Hamburg, Germany \\ ${ }^{2}$ The Hamburg Centre for Ultrafast Imaging, Universität Hamburg, Luruper Chaussee 149, 22761 Hamburg, Germany \\ ${ }^{3}$ Department of Electrical Engineering and Computer Science and Research Laboratory of Electronics, \\ Massachusetts Institute of Technology, Cambridge, Massachusetts 02139, USA \\ Author e-mail address: Anne-Laure Calendron anne-laure.calendron@cfel.de
}

\begin{abstract}
The temperature of a high energy pulsed, cryogenic Yb:YAG composite thin-disk amplifier is optimized for bandwidth to enable extraction of pulses $<10 \mathrm{ps}$ in duration for OPCPA pumping.

OCIS codes: (140.3280) Laser amplifiers; (140.3538) Lasers pulsed; (140.3480) Lasers, diode-pumped
\end{abstract}

\section{Introduction}

High energy lasers at high repetition rate are in great demand for various applications such as pumping of optical parametric chirped pulse amplifiers (OPCPA's), material processing or in scientific instruments such as lasers used in pump-probe experiments in FEL facilities. The OPCPA systems are favored for producing the IR output to drive the high harmonic generation (HHG) or other coherent X-ray sources. For scientific applications, a high repetition rate is useful when the count-rate determines the duration of the experiment.

The heat removal of such high energy, high repetition rate laser drivers has been a challenge; the thin-disk geometry is one technique providing enhanced cooling [1]. Yb:YAG and Yb:LuAG have successfully proven their high-power ability, with up to $5 \mathrm{~kW}$ reported from a single thin-disk in continuous-wave operation [2]. However, at high energy, parasitic lasing limits the maximum stored energy, hence the scalability. To overcome this effect, a shaped end-cap has been suggested in Ref. 3, rejecting the amplified spontaneous emission (ASE) out of the gain medium.

Operating Yb:YAG at liquid nitrogen temperature has the fundamental advantages relative to room-temperature operation of much better thermo-optic properties and dramatically reduced thermal population of the lower laser level that have led to its excellent performance in amplifiers [4, 5]. However, the generation of short energetic pulses desired for OPCPA pumping and THz generation is limited by Yb:YAG's gain bandwidth at low temperature [6]. A compromise that maintains good thermo-optic and spectroscopic properties at increased bandwidth may be reached by raising the temperature to $130-150 \mathrm{~K}$ in the intermediate amplifier stage(s).

In this summary, we are reporting on the characterization of both temperature and bandwidth of a cryogenic Yb:YAG composite thin-disk laser amplifier.

\section{Experimental setup and results}

The laser-head was based on a multipass amplifier constituted of the composite thin-disc (CTD) and a telescope for relay-imaging the beam, ensuring a high beam quality after amplification, as demonstrated first in [7]. The setup is shown on Figure 1 with photos. In the relay-imaging telescope, a spatial filter composed of a small aperture at the focus was employed to clean up the spatial distortions. Due to the flat-top spatial distribution of the pump, the amplified beam acquired after a few passes a smooth super-gaussian beam profile. This is of advantage for later pumping of OPCPA's. First, in order to characterize the extractable power from the CTD, the telescope for the relay imaging was removed and a multi-mode cavity, consisting of the CTD, a $300 \mathrm{~mm}$ focusing lens and an output coupler, was built. The available output couplers were $7.5 \%, 10 \%$ and $15 \%$.

The measurement of the efficiency obtained with the different output couplers is shown on Figure 2 (left). Here, the pump laser diode was set in quasi-continuous-wave operation mode, at $10 \mathrm{~Hz}$ repetition rate, and the pulse duration was varied from $1 \mathrm{~ms}$ to $30 \mathrm{~ms}$, corresponding to an average pump power from $5 \mathrm{~W}$ to $150 \mathrm{~W}$. The crystal temperature was kept constant between $82 \mathrm{~K}$ and $93 \mathrm{~K}$. The slope efficiency was maximum for $10 \%$ output coupling with $52.1 \%$ optical-optical efficiency. Even at the maximal pump power, the efficiency curve was still linear, indicating no limitation from thermal effects or ASE. With $150 \mathrm{~W}$ of pump power, the highest extracted output 
power was nearly $80 \mathrm{~W}$ with $10 \%$ output coupling and dropped to $74.8 \mathrm{~W}$ and $70.0 \mathrm{~W}$ for output coupling levels of $7.5 \%$ and $15 \%$ respectively.

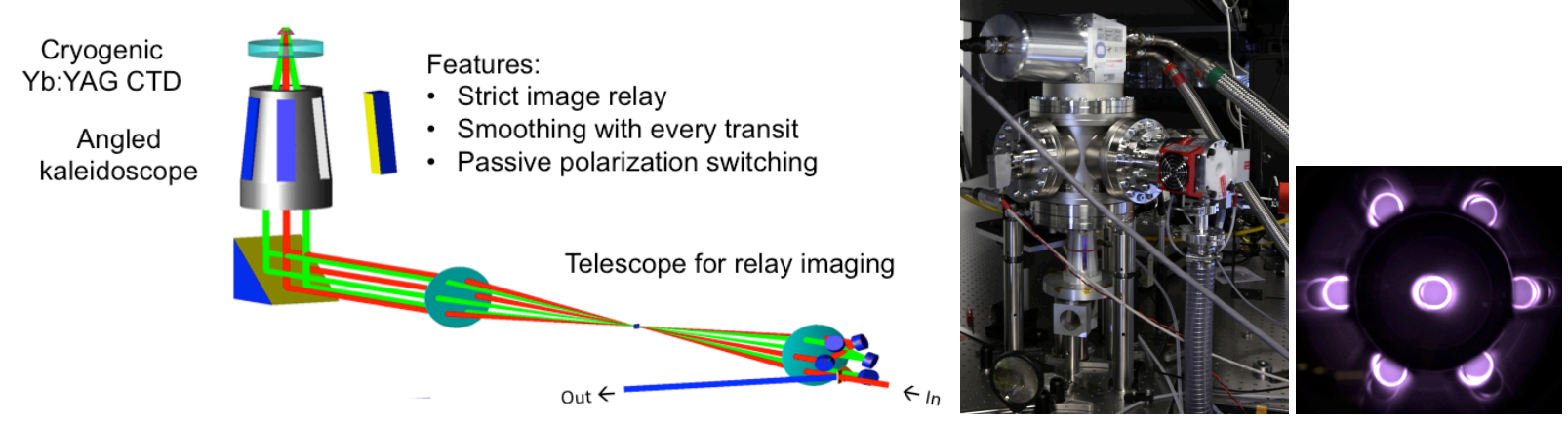

Fig. 1. Layout of the multi-pass amplifier (left), showing the cryogenic composite thin-disc, the kaleidoscope and the relayimaging telescope with spatial filter through a pin-hole. Middle: Photo of the cooling head with the vacuum chamber.

Right: Photo of the pumped thin-disc with reflections on the kaleidoscope mirrors.

On the right side of Figure 2, the dependency of the output power with the crystal temperature is shown. As expected, due to the decrease of the absorption and emission cross-sections with the temperature increase, the output power decreased from $79.9 \mathrm{~W}$ to $23.8 \mathrm{~W}$.
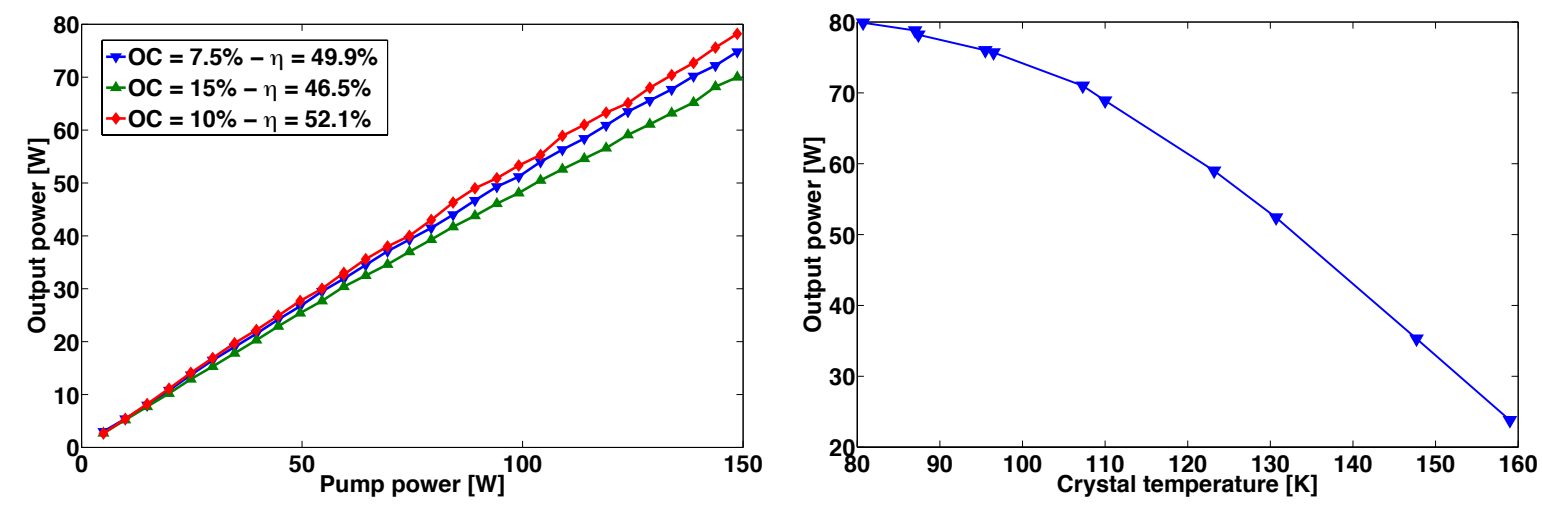

Fig. 2. Power efficiency with variation of the output coupling (left) and dependency of the output power on the crystal temperature (right).

The measurements of the spectral bandwidth were realized with the amplification of $1 \mathrm{~mJ}, 1 \mathrm{kHz}, 1030 \mathrm{~nm}$ pulses out of a commercial regenerative amplifier (Amplitude System s-pulse HP2 custom) seeded with a homemade fiber oscillator. The pulses were stretched to $200 \mathrm{ps}$ and their spectrum supported pulses below 500 fs. By increasing the average pump power, the population inversion in the crystal was increased, thus the gain per pass was higher. The left graph on Figure 3 displays the calculations of the available bandwidth at $77 \mathrm{~K}$ and $130 \mathrm{~K}$, with the measured data points. The measurement was in good agreement with our calculation. The available spectral bandwidth is $0.6 \mathrm{~nm}$ for saturated gain at $77 \mathrm{~K}$.

On the right graph, the normalized spectrum was taken for different pump powers. As a reference, the spectrum before the multipass amplifier is also shown. Above $200 \mathrm{~W}$ of pump power, the spectral bandwidth was narrowed down to $0.75 \mathrm{~nm}$ and remained constant for higher pump power. The central wavelength was $1028.9 \mathrm{~nm}$ independently of the pump power. The amplified spectrum supported Fourier-transform limited pulses below 2 ps. After 12 passes amplification, the energy reached $50 \mathrm{~mJ}$ per pulse at $100 \mathrm{~Hz}$ repetition rate.

At the time of this writing a Helium refrigerator fitted with a heater for the precise control of the composite disk temperature has been activated. We plan to extract pulses and collect bandwidth versus temperature data for extracted pulses that will be presented at the time of the conference. 

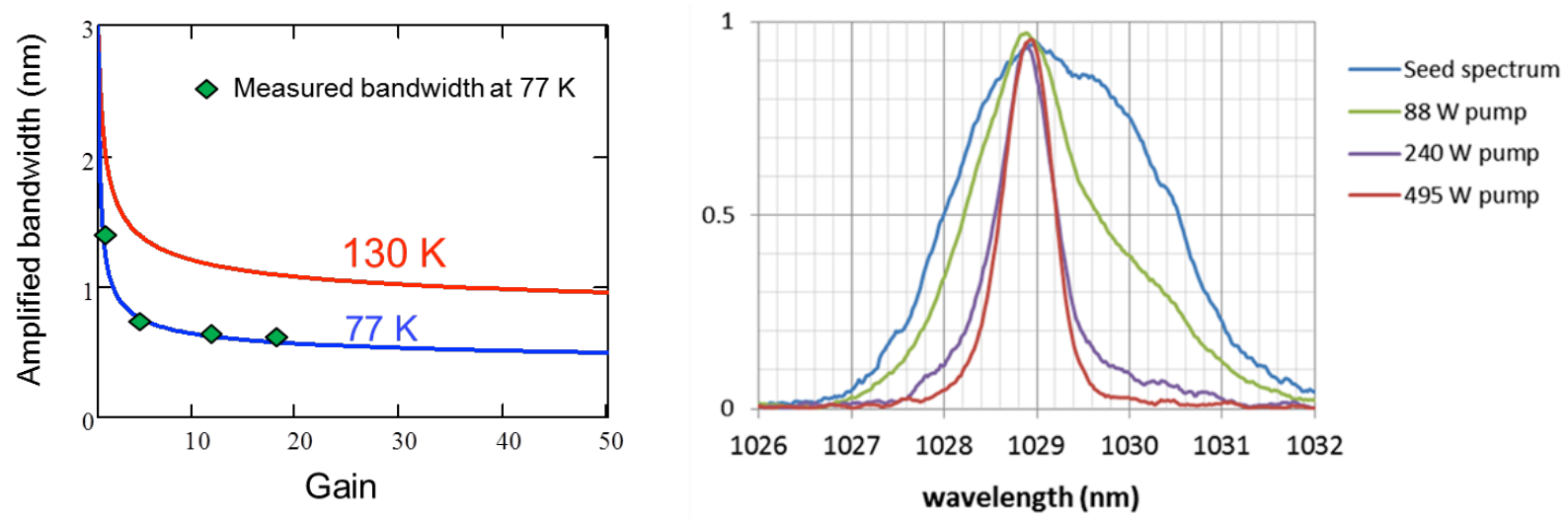

Fig. 2. Study of the influence of the temperature on the bandwidth after amplification. Left: Calculated bandwidth for $77 \mathrm{~K}$ and $130 \mathrm{~K}$ depending on the gain; the green diamond are measured data. Right: Narrowing of the amplified spectrum with the increase of the pump power.

\section{Conclusion}

We demonstrated the operation of the cryogenically cooled composite thin-disk by tuning the temperature and choosing the best compromise between lasing efficiency and bandwidth. The minimal bandwidth, obtained for pump power higher than $200 \mathrm{~W}$ and at $77 \mathrm{~K}$ crystal temperature, was $0.6 \mathrm{~nm}$, corresponding to $1.8 \mathrm{ps}$ long transformlimited pulses.

\section{References}

[1] A. Giesen and J. Speiser, "Fifteen Years of Work on Thin-Disk Lasers: Results and Scaling Laws", IEEE J. of Selected Topics in Quantum Electronics, 13 (3), 598-609 (2007).

[2] K. Beil, S. T. Fredrich-Thornton, F. Tellkamp, R. Peters, C. Kränkel, K. Petermann, and Günter Huber, "Thermal and laser properties of $\mathrm{Yb}: \mathrm{LuAG}$ for kW thin disk lasers", Optics Express, 18 (20), 20712-20722 (2010).

[3] L. E. Zapata, "Edge-Facet Pumped, multi-aperture, thin-disk laser geometry for very high Average Power Output Scaling," U. S. Patent 6,834,070 B2 December 21, 2004. Inventor: under DOE contract No. W-7405-ENG-48 with the University of California.

[4] T. Y. Fan, D. J. Ripin, R. L. Aggarwal, J. R. Ochoa, B. Chann, M. Telleman, and J. Spitzberg, "Cryogenic Yb³-doped solid-state lasers," IEEE J. of Selected Topics in Quantum Electronics, 13 (3), 448-459 (2007).

[5] D. C. Brown, J. M. Singley, E. Yager, K. Kowalewski, J. Guelzow, and J. W. Kuper, "Kilowatt class high-power CW Yb:YAG cryogenic laser," Proc. SPIE 6952, 69520K (2008).

[6] K. H. Hong, A. Siddiqui, Jeffrey Moses, Juliet Gopinath, John Hybl, F. Ömer Ilday, Tso Yee Fan, and Franz X. Kärtner, "Generation of 287 W, 5.5 ps pulses at $78 \mathrm{MHz}$ repetition rate from a cryogenically cooled Yb:YAG amplifier seeded by a fiber chirped-pulse amplification system," Opt. Lett. 33, 2473 (2008).

[7] L. E. Zapata, H. Lin, H. Cankaya, A.-L. Calendron, W. Huang, K.-H. Hong, and F. X. Kaertner, "Cryogenic composite thin disk high energy pulsed, high average power, diffraction limited multi-pass amplifier," Advanced Solid State Lasers Conference Proceedings, AF3A (2013). 